\title{
Tyrosine Kinase Inhibitors and Proton Pump Inhibitors: An Evaluation of Treatment Options
}

\author{
Roelof W. F. van Leeuwen ${ }^{1,2}$ - Frank G. A. Jansman ${ }^{3,4} \cdot$ Nicole G. Hunfeld $^{2,5}$ • \\ Robert Peric $^{6}$ - Anna K. L. Reyners ${ }^{7}$ - Alex L. T. Imholz ${ }^{8}$. Jacobus R. B. J. Brouwers ${ }^{4}$ • \\ Joachim G. Aerts ${ }^{6}$ Teun van Gelder ${ }^{2,9} \cdot$ Ron H. J. Mathijssen ${ }^{1}$
}

Published online: 18 January 2017

(C) The Author(s) 2017. This article is published with open access at Springerlink.com

\begin{abstract}
Tyrosine kinase inhibitors (TKIs) have rapidly become an established factor in oncology, and have been shown to be effective in a wide variety of solid and hematologic malignancies. Use of the oral administration route of TKIs offers flexibility and is convenient for the patient; however, despite these advantages, the oral route of administration also causes a highly relevant new problem. Acid-inhibitory drugs, such as proton pump inhibitors (PPIs), increase the intragastric $\mathrm{pH}$, which may subsequently decrease TKI solubility, bioavailability, and treatment efficacy. Clear and practical advice on how to manage PPI use during TKI therapy is currently not available in the literature. Since PPIs are extensively used during TKI therapy, prescribers are presented with a big dilemma as to whether or not to continue the combined treatment, resulting in patients possibly being deprived of optimal therapy. When all pharmacological characteristics and data of either TKIs and PPIs are considered, practical
\end{abstract}

Roelof W. F. van Leeuwen

r.w.f.vanleeuwen@erasmusmc.nl

1 Department of Medical Oncology, Erasmus MC Cancer InstituteErasmus University Medical Center, 's-

Gravendijkwal 230, 3015 CE Rotterdam, The Netherlands

2 Department of Hospital Pharmacy, Erasmus University Medical Center, 's-Gravendijkwal 230, 3015 CE Rotterdam, The Netherlands

3 Department of Clinical Pharmacy, Deventer Hospital, Nico Bolkesteinlaan 75, 7416 SE Deventer, The Netherlands

4 Department of Pharmacotherapy, Epidemiology and Economics, University of Groningen, A. Deusinglaan 1, 9713 AV Groningen, The Netherlands

5 Department of Intensive Care, Erasmus University Medical Center, 's-Gravendijkwal 230, 3015 CE Rotterdam, The Netherlands and safe advice on how to manage this drug combination can be given.

\section{Key Points}

TKIs have become an established factor in oncology but concomitant use of PPIs decrease TKI bioavailability.

Since PPI use is associated with decreased TKI efficacy, prescribers are posed with a great dilemma whether or not to continue the combined treatment.

When all pharmacological characteristics are considered, a practical and safe advice on how to manage this drug combination can be given.

6 Department of Pulmonary Medicine, Erasmus MC Cancer Institute, 's-Gravendijkwal 230, 3015 CE Rotterdam, The Netherlands

7 Department of Medical Oncology, University Medical Center Groningen, A. Deusinglaan 1, 9712 CP Groningen, The Netherlands

8 Department of Medical Oncology, Deventer Hospital, Nico Bolkesteinlaan 75, 7416 SE Deventer, The Netherlands

9 Department of Internal Medicine, Erasmus University Medical Center, 's-Gravendijkwal 230, 3015 CE Rotterdam, The Netherlands 


\section{Introduction}

Tyrosine kinase inhibitors (TKIs) have rapidly become an established factor in daily oncology practice [1], and have been shown to be effective in a wide variety of solid and hematologic malignancies. At present, there are 25 EMAapproved TKIs, and many new TKIs are under investigation [2]. Use of the oral administration route of TKIs offers logistic flexibility and is convenient for the patient [3]; however, despite these advantages, the oral route of administration also causes a highly relevant new problem. For TKIs in particular, the poor and variable bioavailability, together with other variable pharmacokinetic factors, contribute to a significant in- and between-patient variability in plasma levels and exposure [4]. Most importantly, acid-inhibitory drugs, such as proton pump inhibitors (PPIs), increase the intragastric $\mathrm{pH}$, which may decrease the solubility and thereby the biological availability of certain TKIs. Although there are no prospective studies available, some retrospective data clearly showed that PPI use was associated with decreased TKI efficacy [5-7].

Although PPIs are extensively used during anticancer treatment, there is still much controversy on how to manage drug-drug interactions (DDIs) between TKIs and PPIs [8,9]. To address this, guidelines are provided by the FDA and the European Medicines Agency (EMA) that recommend studying the DDI between pH-dependent drugs and PPIs. Accordingly, for some TKIs the effect of a PPI on absorption from the gut is thoroughly investigated, and specific guidelines for the management of such DDIs are provided in the product label [2]. However, for other TKIs (e.g. afatinib, regorafenib, sunitinib, trametinib and vemurafenib), only basic preclinical pharmacokinetic studies have been executed to date and the in vivo effect of PPIs on these compounds remains unknown.

Next to other factors, TKI therapy is associated with a higher risk for gastrointestinal disorders. Therefore, for many cancer patients using TKIs, there is a solid indication for gastroprotection or treatment of gastrointestinal symptoms with PPIs $[8,10]$. Although not all TKIs show a significant DDI with PPIs, indecisive guidelines still present prescribers with a dilemma as to whether or not to continue the combined treatment in individual patients [1].

\section{Unraveling Drug-Drug Interactions between Tyrosine Kinase Inhibitors (TKIs) and Proton Pump Inhibitors (PPIs)}

To appreciate the background of the DDI between TKIs and PPIs, we review theoretical pharmacokinetic and pharmacodynamic principles, as well as known pharmacokinetic DDI studies.

\subsection{TKI Absorption and Intragastric pH}

Although the absorption of TKIs may be influenced by many factors, the major determinant in TKI absorption is the $\mathrm{pH}$-dependent solubility $[1,11]$. Since TKIs are weakly basic, there is an equilibrium between the ionized and nonionized form that is dependent on intragastric $\mathrm{pH}$. At normal acidic intragastric $\mathrm{pH}(\mathrm{pH}$ range $1-2)$, the equilibrium shifts to the ionized form. Since the ionized form has better solubility, TKI absorption from the gastrointestinal tract is optimal at low intragastric $\mathrm{pH}$; however, when the intragastric $\mathrm{pH}$ is elevated (e.g. due to concurrent PPI use), the balance shifts towards the non-ionized form of the drug and solubility and bioavailability may decrease significantly $[1,12]$.

\subsection{PPI Pharmacology}

Besides TKI bioavailability, the pharmacological profile of PPIs is important to consider for the management of DDIs between TKIs and PPIs. PPIs are highly effective acidinhibitory agents and are registered in a once-daily dose for the majority of their indications. Although this dosing strategy is usually effective in controlling gastroesophageal reflux disease, PPIs do not elevate the intragastric $\mathrm{pH}$ over the full 24-h range (see Fig. 1) [13-16]. There are two important explanations for this $24-\mathrm{h}$ variation in acid suppression: (1) the delayed onset of the pharmacological effect of PPIs; and (2) the duration of pharmacological action [16, 17].

For most PPIs, the acid-inhibitory effects (defined by an intragastric $\mathrm{pH}>4$ ) will only be reached $3-4 \mathrm{~h}$ after intake [16-19]. This delayed onset of action is caused predominantly by the use of enteric-coated tablets or capsules. Since PPIs are easily protonated, they are unstable at low (intragastric) $\mathrm{pH}$ and therefore a coating is indicated. Polymer coatings are stable at low intragastric $\mathrm{pH}$, but break down easily at higher intestinal $\mathrm{pH}$. As a result, the PPI is protected against degradation in the stomach and arrives intact in the duodenum where absorption takes place. Furthermore, the mechanism of action of PPIs through inhibiting the hydrogen/potassium adenosine triphosphatase enzyme $(\mathrm{H}+/ \mathrm{K}+$ ATPase $)$ in gastric cells may also cause a further delay of action [20]. The resulting delay of acid-inhibitory effects after administration amounts to an average of 3-4 h (Fig. 1).

Although most PPIs are characterized by a short half-life $\left(t_{1 / 2}\right)$ of approximately $1-2 \mathrm{~h}$, the pharmacodynamic effects on intragastric $\mathrm{pH}$ last much longer because of its irreversible covalent binding to the proton pumps. After 2-3 days of daily use, a steady state in acid inhibition is reached $[13,20]$. Meanwhile, new proton pumps are generated in vivo on a continuous basis, and, subsequently, 


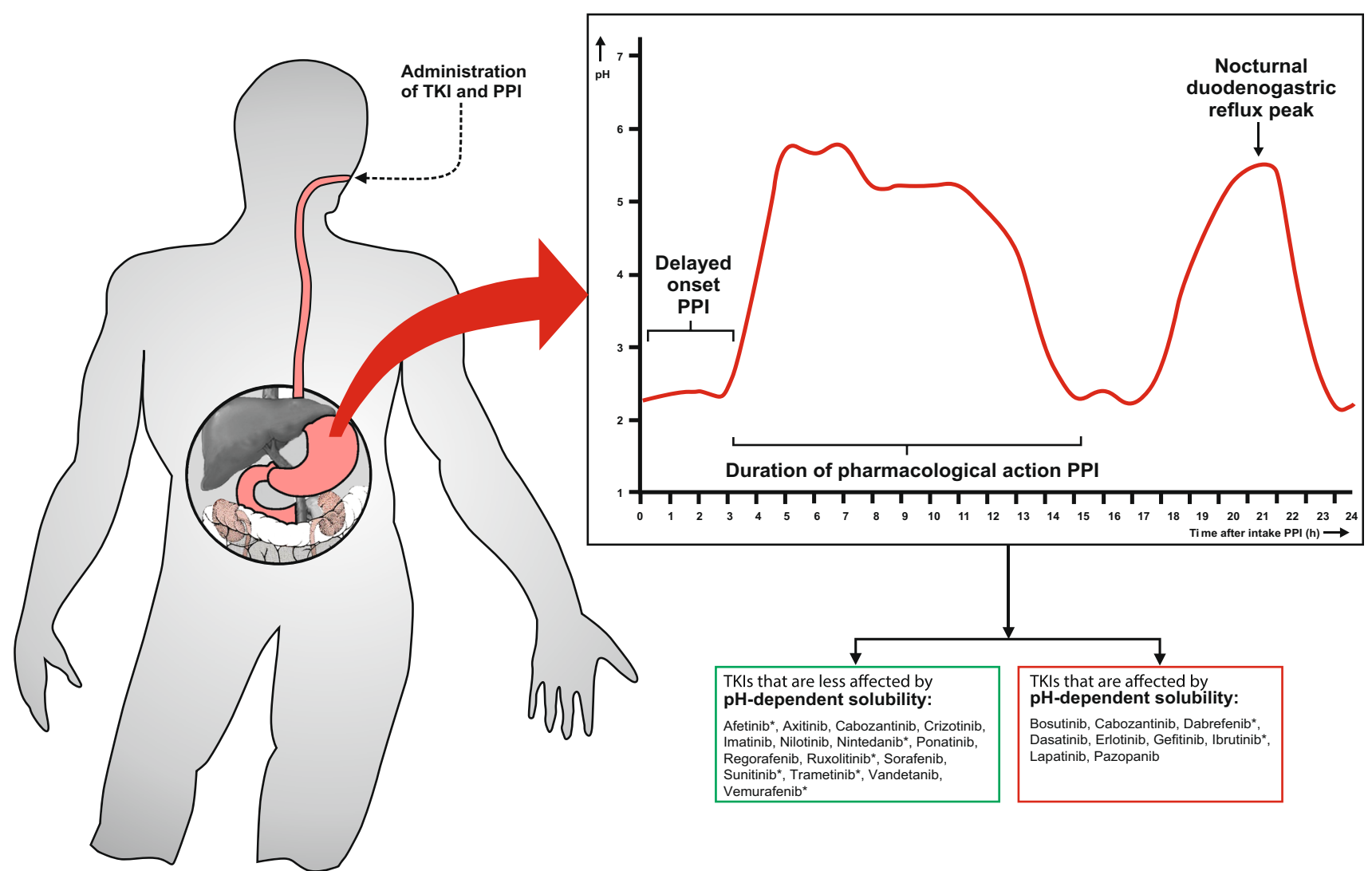

Fig. 1 Schematic 24-h intragastric pH curve during PPI use (entericcoated, once daily) with delayed onset of action (3-4h), duration of action (12-14 h with once-daily use) and the nocturnal duodenogastric reflux peak (obtained by the supine position during sleep).

gastric acid will be secreted from these new pumps, compensating the elevated $\mathrm{pH}$ [16]. As a consequence, the intragastric $\mathrm{pH}$ will start to decrease again and drops to $\mathrm{pH}$ values $<4$ within $12-14 \mathrm{~h}$ after PPI administration (Fig. 1) [16]. On the other hand, during nighttime, physiological duodenogastric reflux occurs as a result of the supine position during sleep. As a result, there is an elevation in intragastric $\mathrm{pH}$ during nighttime which sharply returns to baseline after getting out of bed (Fig. 1) [16]. Furthermore, a substantial proportion of patients above 80 years of age experience achlorhydria, a state in which the production of hydrochloric acid in the stomach is low or absent and the intragastric $\mathrm{pH}$ is substantially elevated [21]. Both nighttime duodenogastric reflux and achlorhydria in older patients may profoundly alter TKI bioavailability. Moreover, TKI bioavailability can be profoundly influenced when taken with food (e.g. lapatinib) [22]. Food may significantly enhance TKI bioavailability by elevating intragastric $\mathrm{pH}$ and drug absorption, resulting in high intra- and interpatient variability. For this reason, patients are often advised to take a TKI in a fasting state. Of note, in serious gastroesophageal reflux disease, physicians may prescribe a PPI in a twice-daily dose. In contrast to a once-daily dose,

Derived from Hunfeld et al. [16], with permission. *Based on in vitro preclinical studies only. TKI tyrosine kinase inhibitor, PPI proton pump inhibitor

more frequent dosing of PPIs (e.g. twice-daily or continuous dosing) leads to a greater and more constant elevation of intragastric $\mathrm{pH}$ over the full 24-h range [10].

\subsection{Available Drug-Drug Interaction Studies and Study Design}

As mentioned in Sect. 2.2, the intragastric $\mathrm{pH}$ is not elevated over the full 24-h range during PPI therapy. Therefore, the outcomes of DDI studies between a PPI and a TKI are highly dependent on the study design, especially the time of intake for both the TKI and the PPI. Two types of studies can be distinguished: (1) the TKI and PPI are administered concomitantly; and (2) the TKI is administered $2-3 \mathrm{~h}$ after the intake of the PPI. There are strengths and limitations for both types of study designs.

When the drugs are taken concomitantly, and if the observed effect is low/nihil, this may indicate that there is indeed no interaction between the two drugs, but it may also well be that a DDI would have been observed if the PPI had been taken at another time [2, 23]. As mentioned previously, when the TKI and PPI are administered concomitantly, there is a time window of low intragastric $\mathrm{pH}$ 
after PPI intake in which the TKI absorption will not be significantly affected, which may potentially lead to a false perception that, regardless of the time of intake of either the PPI and TKI, no DDI occurs. For some TKIs (e.g. axitinib and nilotinib [2]), the question remains whether an alternative time schedule of PPI to TKI intake would lead to an increase or decrease in the TKI absorption, as this is unfortunately rarely studied. Therefore, if no clinically relevant effect is seen in studies while these drugs are taken at the same time, the subsequently drawn conclusion that 'TKIs and PPIs may be used concomitantly' should, in our opinion, be replaced by 'TKIs and PPIs must be used concomitantly', to guarantee safe use.

When a TKI is administered a few hours after a PPI, the intragastric $\mathrm{pH}$ is almost certainly elevated. When no $\mathrm{pH}-$ dependent solubility is expected, a study setup where the TKI is administered a few hours after the PPI might be best in order to completely rule out an absorption-based DDI, as was shown for cabozantinib [24].

\subsection{Management of TKI-PPI Drug Interactions}

There is often a hard indication for concomitant use of TKIs and PPIs; however, in clinical practice, clinicians are often advised to avoid the combination [13], often resulting in the patient being deprived of optimal therapy for gastroesophageal reflux disease.

For several TKIs approved by the FDA, the effect on bioavailability has only been studied in vitro, whereas $\mathrm{pH}$ dependent solubility and TKI absorption in vivo is often multifactorial [4]. In this case, only preclinical in vitro data on chemical $\mathrm{pH}$-dependent solubility may not predict the true in vivo effects on bioavailability (e.g. afatinib, regorafenib, sunitinib, trametinib and vemurafenib [2]) of concomitantly used PPIs. If it is stated that there is no significant DDI between a certain TKI and PPI, this should be confirmed in an adequately designed in vivo pharmacokinetic DDI study, or should be based on population pharmacokinetics for DDI assessment using data from large clinical trials.

A lot of discussion has taken place as to whether TKIs and PPIs are really incompatible. There is the interesting suggestion by Ter Heine et al. that when the PPI dose (in this case pantoprazole) is relatively low, erlotinib can be used concomitantly. However, this recommendation is based on a single case study, and solid pharmacokinetic data provided in the FDA assessment report stated otherwise (mentioning a 46 and $61 \%$ decrease in erlotinib area under the concentration-time curve [AUC] and maximum concentration $\left[C_{\max }\right]$, respectively) $[2,25]$. Although many pharmacokinetic DDI studies have already been conducted and have been published in either an EMA/FDA assessment report or scientific literature, clear advice on the management of the DDI between PPIs and TKIs is rarely given. Studies on alternative time schedules of PPI to TKI intake to completely rule out a DDI are also scarcely available, and drawn conclusions on the management (e.g. can be used concomitantly) may not always be $100 \%$ solid. Clearly, the investigated effect of a PPI on a certain TKI should always be placed in the context of clinical relevance. For instance, if the effect of a PPI on TKI bioavailability does not influence drug efficacy (e.g. $\leq 20 \%$ decrease), this DDI should be considered as nonsignificant.

Due to the nocturnal duodenogastric reflux peak, intragastric $\mathrm{pH}$ is elevated during sleep $[16,26]$. Because of this, the advice to take a TKI without food in the evening concomitantly with a PPI, as was stated in the label of pazopanib, may significantly influence TKI bioavailability [2]. On theoretical grounds (and regardless of PPI use), the bioavailability of TKIs may not be optimal when taken ante noctem and should be avoided accordingly. Furthermore, patients receiving TKI therapy, especially those 80 years of age and older, might experience achlorhydria with a suboptimal absorption as a result [21]. More research is needed to investigate TKI bioavailability during nighttime sleep, and achlorhydria. Although it may significantly enhance TKI bioavailability, patients are often advised to take a TKI without food. As a result, the management of the DDI between the PPI and TKI with regard to food intake $(2 \mathrm{~h}$ before and $1 \mathrm{~h}$ after TKI intake), in clinical practice, also appears to be challenging. When using pantoprazole instead of other PPIs, TKI pharmacokinetics may be altered through inhibition of drug transporters such as Breast Cancer Resistance Protein (BCRP) and P-glycoprotein (Pgp) [27]. Since many TKIs are substrates for BCRP and/or P-gp, physicians should prescribe pantoprazole with caution, or switch to other PPIs, such as omeprazole, during TKI therapy. Moreover, for these TKIs, results obtained from drug interaction studies with omeprazole may not be extrapolated directly to pantoprazole. More research is needed to explore the clinical significance of the DDI between pantoprazole and TKIs.

Several studies have shown that there is large interpatient variability in the onset of action of PPIs. Moreover, there is also large variability in the onset of action between different PPIs and between brand and generic formulas (inter-PPI variability) [16, 17, 28, 29]. Due to these factors, in theory, the delayed onset of action may be significantly shorter. To completely rule out any interpatient and interPPI variability, and to give suitable advice for the management of the DDI between TKIs and all PPIs, TKIs should be taken $2 \mathrm{~h}$ before the PPI. Furthermore, PPIs can be administered either as an enteric-coated (e.g. Losec ${ }^{\circledR}$ or Nexium ${ }^{\circledR}$ ) or instant-release formula (e.g. Zegerid ${ }^{\circledR}$ ). Since the abovementioned delayed onset of action and 
subsequent window of low intragastric $\mathrm{pH}$ is used to manage the DDI between TKIs and PPIs, only the entericcoated formula should be used.

We recently showed that the intake of erlotinib with an acidic beverage (cola) enhanced bioavailability by almost $40 \%$ in patients also taking esomeprazole [30]. Through temporarily lowering the intragastric $\mathrm{pH}$ by administering the TKI with cola, the DDI between TKIs and PPIs can be bypassed (partly). In particular, when there is a hard indication for twice-daily use of a PPI, in our opinion cola may be a simple and practical solution to manage the DDI between TKIs and PPIs.

When all pharmacological characteristics and data of either TKIs and PPIs are considered, balanced, practical and safe advice on how to manage this drug combination can be given. Since the intragastric $\mathrm{pH}$ is not elevated over the whole 24-h range (as shown in Fig. 1), a target period of low intragastric $\mathrm{pH}$ can be used to safely administer the TKI.

\section{Conclusions}

Clinicians and pharmacists should always first assess whether or not there is a clinically relevant DDI between a certain TKI and PPIs. To properly manage a significant DDI between TKIs and PPIs, a twice-daily PPI dose must first be brought back to a once-daily regimen, whereas the PPI must be administered in an enteric-coated formulation. If the TKI is administered in the morning, $2 \mathrm{~h}$ prior to intake of the PPI, the enteric coating of the PPI will provide a target period of low intragastric $\mathrm{pH}$ during which TKIs with a pH-dependent solubility can pass through the stomach with sufficiently low $\mathrm{pH}$. More research on alternative timing schedules of PPI to TKI intake, achlorhydria, and nighttime TKI absorption is necessary as this will provide further insights into the effects of elevated intragastric $\mathrm{pH}$ on TKI bioavailability.

Acknowledgements The authors thank Hans Kneefel for the graphical design of Fig. 1.

\section{Compliance with Ethical Standards}

Funding No funding was provided for the preparation of this article.

Conflict of interest Roelof W. F. van Leeuwen, Frank G. A. Jansman, Nicole G. Hunfeld, Robert Peric, Anna K. L. Reyners, Alex L. T. Imholz, Jacobus R. B. J. Brouwers, Joachim G. Aerts, Teun van Gelder, and Ron H. J. Mathijssen declare no conflicts of interest.

Open Access This article is distributed under the terms of the Creative Commons Attribution-NonCommercial 4.0 International License (http://creativecommons.org/licenses/by-nc/4.0/), which permits any noncommercial use, distribution, and reproduction in any medium, provided you give appropriate credit to the original author(s) and the source, provide a link to the Creative Commons license, and indicate if changes were made.

\section{References}

1. van Leeuwen RW, van Gelder T, Mathijssen RH, Jansman FG. Drug-drug interactions with tyrosine-kinase inhibitors: a clinical perspective. Lancet Oncol. 2014;15(8):e315-26.

2. European Medicines Agency. Europian Public Assessment Reports Assessment History and Product information. http:// www.ema.europa.eu/ema/index.jsp?curl=pages/medicines/landing/ epar_search.jsp\&mid=WC0b01ac058001d124. Accessed 19 Sep 2016.

3. Aisner J. Overview of the changing paradigm in cancer treatment: oral chemotherapy. Am J Health Syst Pharm. 2007;64(9 Suppl 5):S4-7.

4. Herbrink M, Nuijen B, Schellens JH, Beijnen JH. Variability in bioavailability of small molecular tyrosine kinase inhibitors. Cancer Treat Rev. 2015;41(5):412-22.

5. Chu MP, Ghosh S, Chambers CR, Basappa N, Butts CA, Chu Q, et al. Gastric acid suppression is associated with decreased erlotinib efficacy in non-small-cell lung cancer. Clin Lung Cancer. 2015;16(1):33-9.

6. Mir O, Lia M, Litière S, Le Cesne A, Sleijfer S, Leahy M, et al. Impact on outcome of concomitant administration of gastric acid suppression (GAS) therapy and pazopanib in soft tissue sarcoma (STS) patients treated within EORTC 62043/62072 trials. CTOS 2016 (\#2562838). https://www.ctos.org. Accessed 17 Oct 2016.

7. Ha VH, Ngo M, Chu MP, Ghosh S, Sawyer MB, Chambers CR. Does gastric acid suppression affect sunitinib efficacy in patients with advanced or metastatic renal cell cancer? J Oncol Pharm Pract. 2015;21(3):194-200.

8. Smelick GS, Heffron TP, Chu L, Dean B, West DA, Duvall SL, et al. Prevalence of acid-reducing agents (ARA) in cancer populations and ARA drug-drug interaction potential for molecular targeted agents in clinical development. Mol Pharm. 2013;10(11):4055-62.

9. Oude Munnink TH, Schouwink JH, Colen HB, Movig KL. Erlotinib and gastric acid-reducing agents: a combination to avoid or to support? Clin Pharmacol Ther. 2014;96(6):658.

10. Targownik LE, Metge C, Roos L, Leung S. The prevalence of and the clinical and demographic characteristics associated with high-intensity proton pump inhibitor use. Am J Gastroenterol. 2007;102(5):942-50.

11. Budha NR, Frymoyer A, Smelick GS, Jin JY, Yago MR, Dresser $\mathrm{MJ}$, et al. Drug absorption interactions between oral targeted anticancer agents and PPIs: is pH-dependent solubility the Achilles heel of targeted therapy? Clin Pharmacol Ther. 2012;92(2):203-13.

12. Mathijssen RH, Sparreboom A, Verweij J. Determining the optimal dose in the development of anticancer agents. Nat Rev Clin Oncol. 2014;11(5):272-81.

13. Savarino V, Di Mario F, Scarpignato C. Proton pump inhibitors in GORD. An overview of their pharmacology, efficacy and safety. Pharmacol Res. 2009;59(3):135-53.

14. Andersson T, Rohss K, Bredberg E, Hassan-Alin M. Pharmacokinetics and pharmacodynamics of esomeprazole, the $\mathrm{S}$-isomer of omeprazole. Aliment Pharmacol Ther. 2001;15(10):1563-9.

15. Junghard O, Hassan-Alin M, Hasselgren G. The effect of the area under the plasma concentration vs time curve and the maximum plasma concentration of esomeprazole on intragastric $\mathrm{pH}$. Eur $\mathbf{J}$ Clin Pharmacol. 2002;58(7):453-8.

16. Hunfeld NG, Touw DJ, Mathot RA, van Schaik RH, Kuipers EJ. A comparison of the acid-inhibitory effects of esomeprazole and 
rabeprazole in relation to pharmacokinetics and CYP2C19 polymorphism. Aliment Pharmacol Ther. 2012;35(7):810-8.

17. Hunfeld NG, Touw DJ, Mathot RA, Mulder PG, Van Schaik RH, Kuipers EJ, et al. A comparison of the acid-inhibitory effects of esomeprazole and pantoprazole in relation to pharmacokinetics and CYP2C19 polymorphism. Aliment Pharmacol Ther. 2010;31(1):150-9.

18. Bell NJ, Burget D, Howden CW, Wilkinson J, Hunt RH. Appropriate acid suppression for the management of gastro-oesophageal reflux disease. Digestion. 1992;51(Suppl 1):59-67.

19. Howden CW, Burget DW, Hunt RH. Appropriate acid suppression for optimal healing of duodenal ulcer and gastro-oesophageal reflux disease. Scand J Gastroenterol Suppl. 1994;201:79-82.

20. Sachs G, Shin JM, Briving C, Wallmark B, Hersey S. The pharmacology of the gastric acid pump: the $\mathrm{H}+, \mathrm{K}+$ ATPase. Annu Rev Pharmacol Toxicol. 1995;35:277-305.

21. Jansen PA, Brouwers JR. Clinical pharmacology in old persons. Scientifica (Cairo). 2012;2012:723678.

22. Willemsen AE, Lubberman FJ, Tol J, Gerritsen WR, van Herpen CM, van Erp NP. Effect of food and acid-reducing agents on the absorption of oral targeted therapies in solid tumors. Drug Discov Today. 2016;21(6):962-76.

23. Johansson S, Read J, Oliver S, Steinberg M, Li Y, Lisbon E, et al. Pharmacokinetic evaluations of the co-administrations of vandetanib and metformin, digoxin, midazolam, omeprazole or ranitidine. Clin Pharmacokinet. 2014;53(9):837-47.
24. Nguyen L, Holland J, Mamelok R, Laberge MK, Grenier J, Swearingen D, et al. Evaluation of the effect of food and gastric $\mathrm{pH}$ on the single-dose pharmacokinetics of cabozantinib in healthy adult subjects. J Clin Pharmacol. 2015;55(11):1293-302.

25. Ter Heine R, Fanggiday JC, Lankheet NA, Beijnen JH, Van Der Westerlaken MM, Staaks GH, et al. Erlotinib and pantoprazole: a relevant interaction or not? $\mathrm{Br} \mathrm{J}$ Clin Pharmacol. 2010;70(6):908-11.

26. Barlow AP, Hinder RA, DeMeester TR, Fuchs K. Twenty-fourhour gastric luminal $\mathrm{pH}$ in normal subjects: influence of probe position, food, posture, and duodenogastric reflux. Am J Gastroenterol. 1994;89(11):2006-10.

27. Oostendorp RL, Buckle T, Beijnen JH, van Tellingen O, Schellens JH. The effect of P-gp (Mdr1a/1b), BCRP (Bcrp1) and P-gp/BCRP inhibitors on the in vivo absorption, distribution, metabolism and excretion of imatinib. Invest New Drugs. 2009;27(1):31-40.

28. Shimatani T, Inoue M, Kuroiwa T, Xu J, Mieno H, Tazuma S. Acid-suppressive effects of generic omeprazole: comparison of three brands of generic omeprazole with original omeprazole. Dig Liver Dis. 2006;38(8):554-9.

29. Stedman CA, Barclay ML. Review article: comparison of the pharmacokinetics, acid suppression and efficacy of proton pump inhibitors. Aliment Pharmacol Ther. 2000;14(8):963-78.

30. van Leeuwen RW, Peric R, Hussaarts KG, Kienhuis E, IJzerman NS, de Bruijn P, et al. Influence of the acidic beverage cola on the absorption of erlotinib in patients with non-small-cell lung cancer. J Clin Oncol. 2016;34(12):1309-14. 